\title{
Erratum: Inherited Thrombophilia and Pregnancy Complications: Should We Test?
}

\author{
Deepa R.J. Arachchillage, MD ${ }^{1,2}$ Mike Makris, MD ${ }^{3,4}$
}

\footnotetext{
1 Department of Haematology, Imperial College Healthcare NHS Trust and Imperial College London, Hammersmith Hospital, London, United Kingdom

2 Department of Haematology, Royal Brompton Hospital, London, United Kingdom

${ }^{3}$ Sheffield Haemophilia and Thrombosis Centre, Royal Hallamshire Hospital, Sheffield, United Kingdom

${ }^{4}$ Department of Infection, Immunity and Cardiovascular Disease, University of Sheffield, Sheffield, United Kingdom
}

Address for correspondence Deepa R.J. Arachchillage, Department of Haematology, Imperial College Healthcare NHS Trust and Imperial College London, Hammersmith Hospital, 4th Floor, Commonwealth Building, Du Cane Road, London W12 ONN, United Kingdom (e-mail: d.arachchillage@imperial.ac.uk).

Semin Thromb Hemost 2019;45:e1.

ERRATUM

The Editor-in-Chief has informed the publisher that the expansion for "HAPPY" was incorrect in the above article in Seminars in Thrombosis and Hemostasis published on June 4, 2018 (DOI: 10.1055/s-0038-1657782).

The correct expansion is Heparin in pregnant women with Adverse Pregnancy outcome to improve the rate of successful PregnancY.

Issue Theme Editorial Compilation VI; Guest Editors: Emmanuel J. Favaloro, PhD, FFSc (RCPA), and Giuseppe Lippi, MD.
Copyright $\odot 2019$ by Thieme Medical Publishers, Inc., 333 Seventh Avenue, New York, NY 10001, USA. Tel: +1(212) 584-4662.
DOI https://doi.org/ 10.1055/s-0039-1678545. ISSN 0094-6176. 